\title{
The emission polarization of RX J1856.5-3754
}

\author{
N. Chkheidze
}

Tbilisi State University, Chavchavadze Avenue 3, 0128, Tbilisi, Georgia

e-mail: n.chkheidze@gmail.com

Received 12 January 2009 / Accepted 18 March 2009

ABSTRACT

\begin{abstract}
The polarization properties of RX J1856.5-3754 are investigated, based on the plasma emission model presented in previous works. It is shown that if the emission of this source is generated by the synchrotron mechanism, then the X-ray and the optical emission should be linearly polarized and the corresponding degrees of linear polarization are calculated. The change of the position angle through the observable period is investigated. According to the model, the position angle should undergo a full-circle rotation more than once within this period. This unusual behaviour of the position angle is a prediction of the model and its detection would be a strong argument in favour of it. The measurement of the polarization patterns of this source appears to be the most reliable way to make clear its real emission nature.
\end{abstract}

Key words. stars: pulsars: individual: RX J1856.5-3754 - radiation mechanisms: non-thermal - polarization

\section{Introduction}

RX J1856.5-3754 (or RXJ1856, hereafter) is one of the brightest nearby isolated neutron stars discovered by ROSAT (Walter et al. 1996) as an X-ray source and a large number of papers have been devoted to explaining its nature. Its soft X-ray spectrum can be well fit by the Planckian spectrum with a temperature $k T_{\mathrm{bb}}^{\infty} \simeq 63 \pm 3 \mathrm{eV}$ (Burwitz et al. 2003). Thus it is supposed that the emission of this source has a thermal nature. However, the existing models based on this original assumption face problems.

The lack of any significant spectral lines in the X-ray spectrum of RXJ1856 argues against heavy element atmosphere models (Burwitz et al. 2001, 2003), whereas single temperature hydrogen atmosphere fits over-predict the optical flux by a large factor (Pavlov et al. 1996; Pons et al. 2002; Burwitz et al. 2003). As none of the classic models of neutron star atmospheres are able to explain the observed X-ray spectrum, which is well fitted by a simple blackbody model (Burwitz et al. 2003), it has been proposed that the star has no atmosphere but rather has a condensed matter surface (Burwitz et al. 2001; Turolla et al. 2004). This surface might emit a virtually featureless blackbodylike spectrum (as originally proposed by Pavlov 2000). Since the broadband spectra of this source cannot be fitted by a single Planckian spectrum, it is often described by two-temperature blackbody models (Pons et al. 2002; Pavlov et al. 2002; Burwitz et al. 2003). However, condensation of surface matter requires very specific conditions to be fulfilled (Lai \& Salpeter 1997; Lai 2001). Even if these conditions are satisfied, the formation of a non-uniform distribution of the surface temperature (twotemperature blackbody models) still remains unclear. The most adequate fits of the spectra give models which assume that the star has a thin hydrogen atmosphere superposed on a condensed matter surface (Motch et al. 2003; Ho et al. 2007). However, the origin of such thin hydrogen layers fitting the data is a problematic issue.

Alternatively, the observational properties of RXJ1856 can be explained in the framework of the plasma emission model presented in Chkheidze \& Machabeli (2007) and
Chkheidze \& Lomiashvili (2008), which are based on welldeveloped theory of pulsars. This model suggests that the emission from this object is generated by a synchrotron mechanism, switched on as the result of acquirement of pitch angles by relativistic electrons during the quasi-linear stage of the cyclotron instability. The model gives successful fits for broadband spectra, without facing problems typical of the thermal radiation models. Considering the case of a nearly aligned rotator (see Fig. 3), it was predicted that the source should have pulsated with a period $P \sim 1$ s (Chkheidze \& Machabeli 2007). However, subsequent XMM-Newton observations of RXJ1856 discovered that its X-ray emission pulsates with a period $P_{\mathrm{obs}}=7.055 \mathrm{~s}$ (Tiengo $\&$ Mereghetti 2007). This has been explained in the framework of the drift wave driven model (Chkheidze \& Lomiashvili 2008). In particular, the real spin period of the pulsar might differ from the observable one, as a consequence of the existence of very low frequency drift waves in the region of generation of the pulsar emission. These waves are not directly observable but result in a periodic change of curvature of the magnetic field lines and, hence, a periodic change of the emission direction with a period of the drift waves assumed to be equal to the observable period (Lomiashvili et al. 2006).

We suppose that the most reliable argument revealing the real emission nature of this source will be its study with polarization instruments. Therefore in the present paper the polarization properties of RXJ1856 are investigated, in the framework of the plasma emission model developed in previous works (Chkheidze \& Machabeli 2007; Chkheidze \& Lomiashvili 2008). Here we describe the emission model in Sect. 2, investigate the emission polarization in Sect. 3, re-estimate the angular parameters provoked by recently obtained results in Sect. 4 , show the behaviour of the position angle using the new values for the angular parameters in Sect. 5 and discuss our results in Sect. 6.

\section{Emission mechanism}

It is well known that the distribution function of relativistic particles is one dimensional at the pulsar surface, because any 
transverse momenta $\left(p_{\perp}\right)$ of relativistic electrons are lost in a very short time $\left(\leq 10^{-20} \mathrm{~s}\right)$ via synchrotron emission in very strong $B \sim 10^{12} \mathrm{G}$ magnetic fields. For typical pulsars, the plasma consists of the following components: the bulk of the plasma with an average Lorentz-factor $\gamma_{\mathrm{p}} \simeq 10$; the tail of the distribution function with $\gamma_{\mathrm{t}} \simeq 10^{4}$ and the primary beam with $\gamma_{\mathrm{b}} \simeq 10^{6}$ (see Fig. 1 from Arons 1981). The generation of waves is possible during the further motion of the relativistic particles along the dipolar magnetic field lines if the condition of cyclotron resonance is fulfilled (Kazbegi et al. 1991):

$\omega-k_{\varphi} V_{\varphi}-k_{x} u_{x}+\omega_{B} / \gamma_{\mathrm{r}}=0$

where $k_{\varphi}^{2}+k_{\perp}^{2}=k^{2}, k_{\perp}^{2}=k_{x}^{2}+k_{r}^{2}, V_{\varphi}$ is the particle velocity along the magnetic field, $\gamma_{\mathrm{r}}$ is the Lorentz factor for the resonant particles, $u_{x}=c V_{\varphi} \gamma_{\mathrm{r}} / \rho \omega_{B}$ is the drift velocity of the particles due to curvature of the field lines, $\rho$ is the radius of curvature of the field lines and $\omega_{B}=e B / m c$ is the cyclotron frequency. Here a cylindrical coordinate system is chosen, with the $x$-axis directed perpendicular to the plane of the field lines, while $r$ and $\varphi$ are the radial and azimuthal coordinates. The reason for the generation of waves is an anisotropy of the distribution function. During the quasi-linear stage of the instability, a diffusion of particles arises not only along but also across the magnetic field lines. Therefore, resonant electrons acquire transverse momenta (pitch angles) and, as a result, start to radiate through the synchrotron mechanism.

In Chkheidze \& Machabeli (2007), it has been assumed that the emission of RXJ1856 is generated by the synchrotron mechanism, which is switched on during the quasi-linear stage of the cyclotron instability (developed at distances $r \sim 10^{9} \mathrm{~cm}$ ). The observed X-ray spectrum is the result of synchrotron radiation of the primary-beam electrons. Particularly, the synchrotron spectrum of the beam electrons with the initial distribution function:

$f_{\| \mathrm{b}}=\frac{n_{\mathrm{b}}}{\sqrt{\pi} \gamma_{\mathrm{T}}} \exp \left[-\frac{\left(\gamma_{\mathrm{r}}-\gamma_{\mathrm{b}}\right)^{2}}{\gamma_{\mathrm{T}}^{2}}\right]$,

matches well the measured $\mathrm{X}$-ray spectrum in the energy interval $(0.26-0.9) \mathrm{keV}$ (here $\gamma_{\mathrm{T}} \simeq 10$ - is the half-width of the distribution function, $n_{\mathrm{b}}=B / P c e$ is the density of primary beam electrons, equal to the Goldreich-Julian density (Goldreich \& Julian $1969)$ and $P$ is the pulsar rotation period). The wave excitation leads to a redistribution of the resonant particles via quasi-linear diffusion. Consequently, after the quasi-linear evolution stage of the instability and by achieving the stationary state the distribution function of the beam electrons takes the following form:

$f_{\| \mathrm{b}} \propto \gamma_{\mathrm{r}}^{-4}$.

But at the same time, the radiation density appears to be sufficiently high and the self-absorption effects begin to play a main role. The synchrotron self-absorption redistributes the emission spectrum in the energy domain of relatively low frequencies and the X-ray emission spectrum corresponding to the energy interval (0.15-0.26) keV has the form of $v^{5 / 2}$ (regardless of the spectrum of the emitting particles prior to absorption processes). The theoretical X-ray spectrum matches the measured one well (see Fig. 4 from Chkheidze \& Machabeli 2007). The observed optical spectrum is the result of the synchrotron emission of the tail electrons with the initial distribution function $f_{\| t_{0}} \propto \gamma^{-4}$. By achieving the stationary state the distribution function of the tail electrons, which is changed due to quasi-linear diffusion has the form:

$f_{\| \mathrm{t}} \propto \gamma_{\mathrm{r}}^{2}$

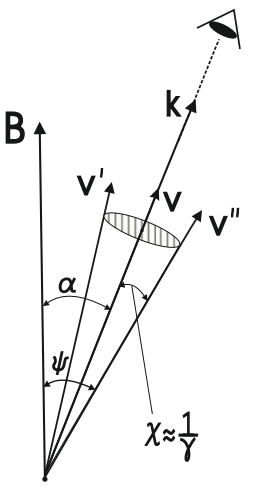

Fig. 1. $\boldsymbol{k}$ is the emission direction, $\boldsymbol{B}$ the magnetic field line, $\boldsymbol{v}, \boldsymbol{v}^{\prime}$ and $v^{\prime \prime}$ are the electron velocity vectors.

The resulting theoretical spectrum of the synchrotron emission of the tail electrons with the distribution function (4) matches the measured one closely.

\section{Emission polarization}

Now let us consider the polarization properties of RXJ1856. For this reason, we have to find the Stokes parameters, which, in our case, are defined as follows (Ginzburg 1981):

$$
\begin{aligned}
I(v)= & \frac{3 e^{3}}{2 \pi} \int B\left(\frac{v}{v_{\mathrm{c}}}\right)^{2}\left(1+\chi^{2} \gamma^{2}\right) \gamma f(\gamma, \psi) \sin \alpha \\
& \times\left[\left(1+\chi^{2} \gamma^{2}\right) K_{2 / 3}^{2}\left(g_{v}\right)+\chi^{2} \gamma^{2} K_{1 / 3}^{2}\left(g_{v}\right)\right] \mathrm{d} \chi \mathrm{d} \gamma \\
Q(v)= & \frac{3 e^{3}}{2 \pi} \int B\left(\frac{v}{v_{\mathrm{c}}}\right)^{2}\left(1+\chi^{2} \gamma^{2}\right) \gamma^{2} f(\gamma, \psi) \sin \alpha \cos 2 \tilde{\chi} \\
& \times\left[\left(1+\chi^{2} \gamma^{2}\right) K_{2 / 3}^{2}\left(g_{v}\right)-\chi^{2} \gamma^{2} K_{1 / 3}^{2}\left(g_{v}\right)\right] \mathrm{d} \chi \mathrm{d} \gamma
\end{aligned}
$$

$$
\begin{aligned}
U(v)= & \frac{3 e^{3}}{2 \pi} \int B\left(\frac{v}{v_{\mathrm{c}}}\right)^{2}\left(1+\chi^{2} \gamma^{2}\right) \gamma^{2} f(\gamma, \psi) \sin \alpha \sin 2 \tilde{\chi} \\
& \times\left[\left(1+\chi^{2} \gamma^{2}\right) K_{2 / 3}^{2}\left(g_{v}\right)-\chi^{2} \gamma^{2} K_{1 / 3}^{2}\left(g_{v}\right)\right] \mathrm{d} \chi \mathrm{d} \gamma
\end{aligned}
$$

$$
V(v)=\frac{3 e^{3}}{\pi} \int B\left(\frac{v}{v_{\mathrm{c}}}\right)^{2}\left(1+\chi^{2} \gamma^{2}\right)^{3 / 2} \gamma^{2} \chi f(\gamma, \psi) \sin \alpha
$$$$
\times K_{1 / 3}\left(g_{v}\right) K_{2 / 3}\left(g_{v}\right) \mathrm{d} \chi \mathrm{d} \gamma \text {, }
$$

where

$$
v_{\mathrm{c}} \approx 4 \times 10^{3} B \gamma^{2}, \quad g_{v}=\frac{v}{2 v_{\mathrm{c}}}\left(1+\chi^{2} \gamma^{2}\right)^{3 / 2},
$$

$f(\gamma, \psi)=f_{\|}(\gamma) f_{\perp}(\psi)$

Here $\alpha$ is the angle between $\boldsymbol{B}$ and $\boldsymbol{k}, \psi$ is the pitch angle and $\chi$ is the angle between the wave and the electron's velocity vectors (see Fig. 1). Also, $\gamma$ is the Lorentz factor of the emitting electrons, $f_{\|}(\gamma)$ and $f_{\perp}(\psi)$ are the distribution functions of the emitting particles from their parallel and perpendicular momenta, respectively, $K_{2 / 3}\left(g_{v}\right)$ and $K_{1 / 3}\left(g_{v}\right)$ are the Macdonald functions and the angle $\tilde{\chi}$ defines the direction of the maximum intensity of the polarized component on the plane of sky.

The expression for the distribution function of the emitting electrons by their perpendicular momenta depends on the value of the following quantity $\psi_{0} \gamma_{\mathrm{r}}$ (where $\psi_{0}$ is the mean value of the 


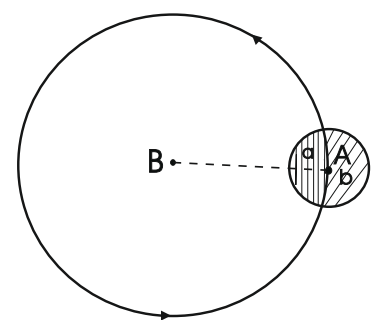

Fig. 2. Point B corresponds to the magnetic field line and point A to the observer's line of sight.

pitch angle). In our case, according to Chkheidze \& Machabeli (2007) the condition $\psi_{0} \gamma_{\mathrm{r}} \gg 1$ is fulfilled for the resonant particles. Then, the emitting particles' distribution function from their pitch angles has the following form (Malov \& Machabeli 2002):

$$
f_{\perp}(\psi) \propto \mathrm{e}^{-A \psi^{4}}, \quad A=\frac{4 e^{6}}{3 \pi^{3} m^{5} c^{7}} \frac{B^{4} P^{3} \gamma_{\mathrm{p}}^{4} \gamma_{\mathrm{r}}^{2}}{\gamma_{\mathrm{b}}^{3}} .
$$

Synchrotron emission of a single electron is strongly beamed along the direction of motion in a cone of approximate opening half-angle $1 / \gamma$. Therefore, in the given direction, the observer detects radiation of electrons whose velocities fill the cone with the opening angle $2 / \gamma$ and the major axis coincides with the line of sight of the observer (see Fig. 1). Hence, at a given moment of time, the observer receives emission of electrons with the pitchangles from the interval $\psi=\alpha+\chi \subset[\alpha-1 / \gamma, \alpha+1 / \gamma]$ (the angle $\chi$ is considered to be positive if $\psi>\alpha$ and negative otherwise). The Stokes parameter $V$ is an odd function of the variable $\chi$, therefore, its integration over a symmetric interval is zero. In the upper panel of Fig. 1 (see Fig. 2), the larger circle represents the cross-section of the cone, the lateral surface of which is described by the electron velocity vector moving on a spiral path along the magnetic field line. The circle with a smaller radius is the cross-section of the cone combined with the electrons' velocity vectors, which provide significant radiation in the observer's direction. The area of the smaller circle is striped in two different ways: area a corresponds to electrons with positive values of the angle $\chi$, while area $\mathbf{b}$ corresponds to those with negative values of the angle $\chi$. If $\mathbf{a} \neq \mathbf{b}$ then $V$ has a non-zero value. But bearing in mind that the radius of the large circle is much greater than the smaller one's radius (the opening angles of the corresponding cones are of the order of $10^{-3}$ and $10^{-6}$ for the beam electrons and $10^{-1}$ and $10^{-4}$ for the tail electrons, respectively), one can assume that $\mathbf{a} \approx \mathbf{b}$. But symmetry of integration bounds alone not enough to have a zero circular polarization. Also, the distribution function of the emitting particles by their pitch angles (11) should vary slowly within the small interval $\chi \subset[-1 / \gamma, 1 / \gamma]$. Estimations show that the latter condition is well fulfilled. Thus, we conclude that the emission of this source is not circularly polarized.

Now we can calculate the degree of linear polarization for the X-ray and optical emission, which is defined as (Ginzburg 1981)

$\Pi_{1}=\frac{\sqrt{Q^{2}+U^{2}}}{I}$.

The degree of linear polarization of the X-ray spectrum, correspondent to the energy interval (0.26-0.9) keV can be calculated from Eqs. (2), (5), (6), (7) and (11), which give $\Pi_{1} \approx(84-79) \%$. As it was mentioned above, the observed X-ray spectrum at relatively low frequencies $(0.15-0.26) \mathrm{keV}$ is produced as the result

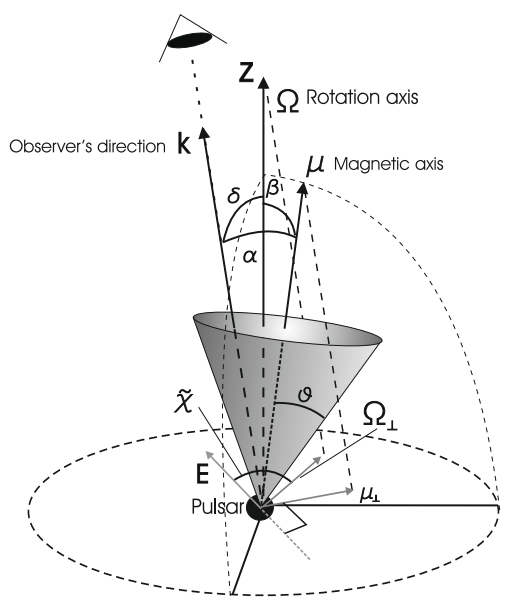

Fig. 3. $\boldsymbol{\Omega}$ is the rotation axis, $\boldsymbol{\mu}$ - the magnetic-moment axis, $\boldsymbol{k}$ - the observer's axis and $\boldsymbol{E}$ is the electric field vector of the radiation. $\boldsymbol{\mu}_{\perp}$ and $\boldsymbol{\Omega}_{\perp}$ are the projections of the corresponding vectors on the plane of sky, $\tilde{\chi}$ is the position angle.

of synchrotron self-absorption processes. The degree of linear polarization in this case is defined as follows:

$\Pi_{1}=\frac{3}{6 d+13}$

where $d=4$, in our case. The calculation gives $\Pi_{1} \approx 8 \%$. So, in the energy interval $(0.15-0.26 \mathrm{keV})$, the degree of linear polarization of the X-ray emission sharply decreases.

The observed optical spectrum is the result of the synchrotron emission of tail electrons with the distribution function (4). The expression (12) yields in this case $\Pi_{1} \approx 86 \%$.

\section{Estimation of the angular parameters}

In previous work (Chkheidze \& Lomiashvili 2008), the values of angular parameters for RXJ1856 have been estimated by fitting the simulated X-ray emission light curve with the observational data (see Fig. 2 from Chkheidze \& Lomiashvili 2008). In this section more accurate fitting is done, taking into account the recently obtained results. This allows us to estimate the angular parameters more exactly.

The observations detect continuous radiation, meaning that the condition $\alpha(t) \leq \vartheta$ is fulfilled at any moment of time (where $\vartheta$ is the opening half-angle of the X-ray emission cone). To estimate the value of $\vartheta$ we use the emitting particles distribution function by their pitch angles. According to Eq. (11), the greatest possible value of the pitch angle, acquired by the beam electron at the quasi-linear stage of the cyclotron instability is of the order of $\psi_{0}\left(\psi_{0} \simeq 10^{-3}\right.$ for the primary-beam electrons, Chkheidze $\&$ Machabeli 2007). The electron emits along the direction of motion through the cone with the opening angle $1 / \gamma$, which for the primary-beam electrons is equal to $10^{-6}$. The magnetic field lines are nearly parallel and straight in the emitting region (as their curvature $\rho \simeq 10^{8} \mathrm{~cm}$ ). Thus, we can conclude that the angular size of the emission cone is equal to the maximal possible value of the pitch angle of the emitting electrons, meaning that the opening angle of the X-ray emission cone $\vartheta \simeq 10^{-3}$.

The emission intensity of the set of electrons in the given direction is defined by Eq. (5). Its time dependence can be approximately expressed in the following form:

$I(t) \propto \sin \alpha(t) \mathrm{e}^{-A \alpha(t)^{4}}$. 


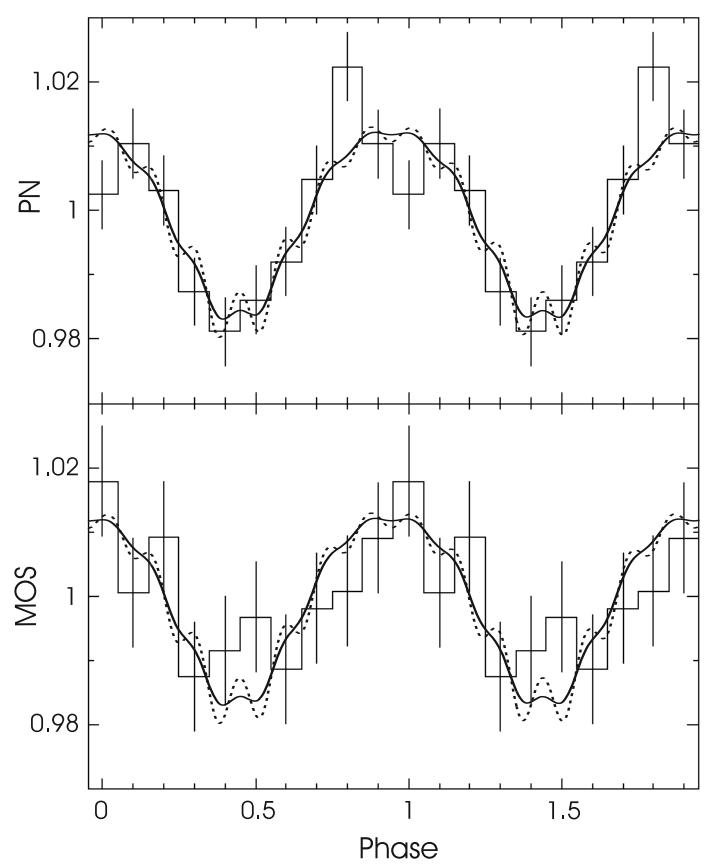

Fig. 4. The simulated light curves of RX J1856.5-3754 fitted with PN and MOS observations. The solid and dotted lines correspond to $\delta \sim$ $10^{-6}$ and $\delta \sim 10^{-5}$, respectively.

Here the angle $\alpha$ has a fixed value at a fixed time moment, but due to the star's rotation it undergoes periodic variations (Lomiashvili et al. 2006):

$\alpha(t)=\arccos [\sin \delta \sin \beta \cos \Omega t+\cos \delta \cos \beta]$.

Here $\delta$ is the angle between the rotation and the observer's axes and $\beta$ is the angle between the rotation and the magneticmomentum axes (see Fig. 2). In the absence of the drift waves, $\beta=\beta_{0}=$ const. and the period of $\alpha$ is equal to $2 \pi / \Omega$ (where $\Omega$ is the angular velocity of the pulsar). But in the presence of the drift waves $\beta=\beta(t)$ is harmonically oscillating about $\beta_{0}$, with an amplitude $\Delta \beta=\Delta \rho / \rho$ and rate $\omega_{\mathrm{dr}}=2 \pi / P_{\mathrm{dr}}$ (here $\Delta \rho$ is the amplitude of change of the field line curvature, $P_{\mathrm{dr}}$ and $\omega_{\mathrm{dr}}$ are the period and the cyclic frequency of the drift waves). Thus we can write that

$\beta(t)=\beta_{0}+\Delta \beta \sin \left(\omega_{\mathrm{dr}} t+\varphi\right)$

Accordingly, for the angle $\alpha$ we will get:

$$
\begin{aligned}
\alpha= & \arccos \left[\sin \delta \sin \left(\beta_{0}+\Delta \beta \sin \left(\omega_{\mathrm{dr}} t+\varphi\right)\right) \cos \Omega t\right. \\
& \left.+\cos \delta \cos \left(\beta_{0}+\Delta \beta \sin \left(\omega_{\mathrm{dr}} t+\varphi\right)\right)\right] .
\end{aligned}
$$

If $\delta \ll 1$, the period of change of $\alpha$ equals $P_{\mathrm{dr}}$ and, in this case, the observable period $P_{\mathrm{obs}}=7.055 \mathrm{~s}$ does not represent the real spin period of the pulsar, which is estimated to be $P \sim 1 \mathrm{~s}$ (Chkheidze \& Lomiashvili 2008).

The fitting is done using the time dependence of the emission intensity obtained in the framework of the model. Two simulated light curves are plotted in Fig. 4 with different values for the angle $\delta$. As we can see, the change of $\delta$ causes the appearance of additional peaks, corresponding to pulsations with a real spin period, which is approximately equal to $1 \mathrm{~s}$. Therefore, we expect that the detection of the "real" spin period of RXJ1856 can be achieved with observations of higher resolution. The new values of the angular parameters (see Table 1) give the fulfillment of the condition $\alpha(t) \leq 10^{-3}$ at any moment of time.

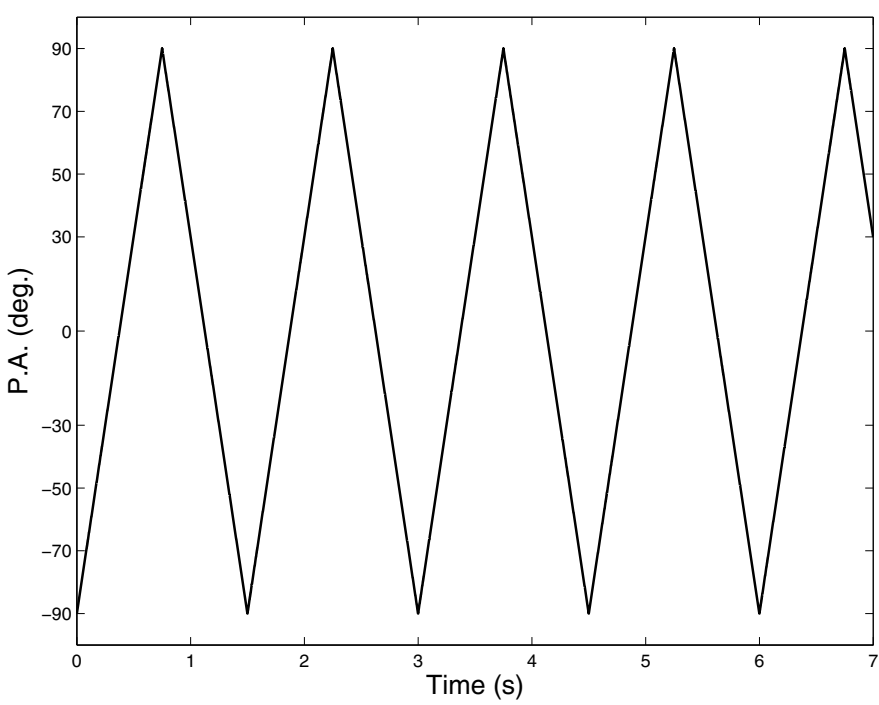

Fig. 5. The behaviour of the position angle of linearly polarized X-ray emission within one observable pulse.

Table 1. The values of angular parameters for RX J1856.5-3754.

\begin{tabular}{llrrrr}
\hline \hline$P_{\text {obs }}(s)$ & $P(s)$ & $\Delta \beta$ & $\beta_{0}$ & $\delta$ & $\vartheta$ \\
\hline 7.055 & 1.5 & $10^{-4}$ & $2 \times 10^{-3}$ & $2 \times 10^{-5}$ & $10^{-3}$ \\
\hline
\end{tabular}

\section{Position angle}

It is very important to know the behaviour of the position angle of linear polarization of the X-ray emission through the observable pulse. If we use the spherical coordinate system $(r, \varphi, \theta)$ (see Fig. 3), then we can write:

$\boldsymbol{k}=(1,0, \delta)$

$\boldsymbol{\mu}=(1, \Omega t, \beta)$,

$\boldsymbol{\Omega}=(1,0,0)$.

The position angle is the angle between the electric field vector of the radiation and the projected spin axis on the plane of sky. From Fig. 3, it is easy to find the expression for PA

$\tilde{\chi}=\frac{\pi}{2}-\arccos \left(\frac{\cos \beta-\cos \alpha \cos \delta}{\sin \alpha \sin \delta}\right)$.

If we assume that the real spin period of RXJ1856 $P=1.5 \mathrm{~s}$, then in $7.055 \mathrm{~s}$ the star should make a full-circle rotation more than once. Consequently, the position angle should also rotate by $360^{\circ}$ a few times within the observable period (see Fig. 5). The detection of such unusual behaviour of the position angle would be the strongest argument for our model.

\section{Discussion}

In previous works (Chkheidze \& Machabeli 2007; Chkheidze \& Lomiashvili 2008) the plasma emission model of RXJ1856 was presented. It is supposed that the emission of this source is generated by the synchrotron mechanism. The distribution function of relativistic particles is one dimensional at the pulsar surface, but plasma with an anisotropic distribution function is unstable which can lead to wave excitation. The main mechanism of wave generation in plasmas of the pulsar magnetosphere is the cyclotron instability. During the quasi-linear 
stage of the instability, a diffusion of particles arises along and across the magnetic field lines. Therefore, plasma particles acquire transverse momenta and, as a result, the synchrotron mechanism is switched on. The measured X-ray and optical spectra are the results of synchrotron emission of primary-beam and tail electrons, respectively. The predictable characteristic frequencies $v_{\mathrm{m}}(\mathrm{X}$-ray $) \simeq 6 \times 10^{16} \mathrm{~Hz}$ and $v_{\mathrm{m}}($ Optic $) \simeq 2 \times 10^{14} \mathrm{~Hz}$ (where $v_{\mathrm{m}}$ is the frequency of the maximum in the synchrotron spectrum) lie in the same domains as the measured spectra.

The original waves, excited during cyclotron resonance, lie in the radio domain, but radio emission is not observed from $\mathrm{RXJ1856.} \mathrm{One} \mathrm{of} \mathrm{the} \mathrm{possible} \mathrm{explanations} \mathrm{as} \mathrm{to} \mathrm{why} \mathrm{the} \mathrm{radio}$ emission is not detected from this object is that the emission traverses a large distance in the pulsar magnetosphere (since the model of a nearly aligned rotator is used). So there is a high probability that the excited waves are in the cyclotron damping range: $\omega-k_{\varphi} V_{\varphi}-k_{x} u_{x}-\omega_{B} / \gamma_{\mathrm{r}}=0$ (Khechinashvili \& Melikidze 1997). In this case, the radio emission will not reach the observer. Nevertheless, the detection of radio emission from RXJ1856 would be a strong argument in favour of the model.

The effectiveness of the cyclotron mechanism has been estimated and it appears to be quite efficient (Chkheidze \& Machabeli 2007). For effective generation of waves it is essential that the time during which the particles give energy to waves should be more than $1 / \Gamma$ (where $\Gamma$ is the growth rate of instability). The radio waves propagate practically in straight lines, whereas the dipolar magnetic field lines deviate from their initial direction, and the angle $\theta=k_{\|} / k_{\varphi}$ grows. $\theta$ is the angle between the wave line and the line of the dipole magnetic field. On the other hand, the resonance condition (1) imposes limitations on $\theta$ i.e. particles can resonate with the waves propagating in a limited range of angles. The estimations show that in our case, the fulfillment of $\rho \gtrsim 3 \times 10^{9} \mathrm{~cm}$ implies an effectiveness of the cyclotron mechanism. As the instability develops at distances $r \sim 10^{9} \mathrm{~cm}$, it follows that the excited waves lie in the resonant region long enough for particles to acquire pitch angles and to generate the observed radiation.

The total energy available for conversion into pulsar emission has been estimated as $\dot{E} \simeq n_{\mathrm{b}_{0}} \pi R_{\mathrm{pc}}^{2} \gamma_{\mathrm{b}} m c^{3}$. Here, $n_{\mathrm{b}_{0}}$ is the Goldreich-Julian density at the pulsar surface and $R_{\mathrm{pc}}$ is the radius of the polar cap. The estimations show $\dot{E} \simeq 5 \times 10^{32} \mathrm{erg} / \mathrm{s}$, which can explain the observed X-ray luminosity of RXJ1856.

The recently discovered $7 \mathrm{~s}$ pulsations of the X-ray emission of RXJ1856 has been explained in the framework of the drift wave driven model. The main feature of this model is that the spin period of the pulsar might differ from the observable period (for RXJ1856 the real spin period is estimated to be $\sim 1 \mathrm{~s}$ ), as a consequence of the existence of very low frequency drift waves in the region of generation of the pulsar emission. These particular waves are not detected but only result in a periodic change of curvature of the magnetic field lines, which in turn cause the change of observed radiation with a period of the drift wave.

In the present paper the emission polarization of RXJ1856 was investigated. It was shown that both the X-ray and the optical emission are linearly polarized. The degree of linear polarization for the $\mathrm{X}$-ray emission is $\Pi_{1} \approx 8 \%$ in the energy interval $(0.15-0.26) \mathrm{keV}$ and $\Pi_{1} \approx(84-79) \%$ in the energy interval $(0.26-0.9) \mathrm{keV}$. The calculation of polarization degree for optical emission yields $\Pi_{1} \approx 86 \%$. The behaviour of the position angle is also investigated. It is shown that within $7 \mathrm{~s}$ it should rotate by $360^{\circ}$ a few times, depending on the exact value of the real spin period. Particularly, in the present paper, the value of $P=1.5 \mathrm{~s}$ is taken as the real spin period of RXJ1856. In this case the position angle makes four full rotations and a turn of $254^{\circ}$ within the observable spin period of $P_{\mathrm{obs}} \approx 7.055 \mathrm{~s}$. The detection of similar behaviour of the PA would be a strong argument in favour of the plasma emission model.

We suppose that the most reliable argument revealing the real emission nature of RXJ1856 will be the study of this object with polarization instruments. If the emission of this source has a thermal nature, then according to Ho (2007) (this model gives the best match of the entire spectrum, among other thermal emission models) the X-ray emission should be linearly polarized with the polarization degree equal to $100 \%$. The position angle should undergo small changes for the case of $\left(2^{\circ}, 30^{\circ}\right)$ and for the case of $\left(30^{\circ}, 2^{\circ}\right)$ it should rotate by $360^{\circ}$ in one observable pulse (where these quantities are the possible angles between the rotation and the magnetic pole axes and between the rotation and the observer's axes). But if the emission of this source is generated by the synchrotron mechanism, it is expected that both the $\mathrm{X}$-ray and the optical emissions will be linearly polarized with the frequency dependent polarization degree, giving the values from a few percent up to $84 \%$. The position angle should rotate by $360^{\circ}$ within one "real" pulse supposed to be of the order of $1 \mathrm{~s}$. It should undergo full circular rotation several times within the observable period of $7.055 \mathrm{~s}$. Also, no periodic variation of the degree of linear polarization should be observed, since both the numerator and the denominator of Eq. (12) have the same time dependence (see Eq. (15)).

Acknowledgements. The author is grateful to George Machabeli for valuable discussions. This work was partially supported by Georgian NSF Grant ST06/4096. N.C. thanks the Abdus Salam International Center for Theoretical Physics in Trieste, Italy.

\section{References}

Arons, J. 1981, in Proc. Varenna Summer School and Workshop on Plasma Astrophysics, ESA, 273

Burwitz, V., Zavlin, V. E., Neuhäuser, R., et al. 2001, A\&A, 379, L35

Burwitz, V., Haberl, F., Neuhäuser, R., et al. 2003, A\&A, 399, 1109

Chkheidze, N., \& Machabeli, G. 2007, A\&A, 471, 599

Chkheidze, N., \& Lomiashvili, D. 2008, NewA, 13, 12

Drake, J. J., Marshall, H. L., Dreizler, S., et al. 2002, ApJ, 572, 996

Ginzburg, V. L. 1981, Teoreticheskaia Fizika i Astrofizika (Moskva: Nauka)

Goldreich, P., \& Julian, W. H. 1969, ApJ, 157, 869

Ho, W. C. G. 2007, MNRAS 380, 71

Ho, W. C. G., Kaplan, D. L., Chang, P., Adelsberg, M., \& Potekhin, A. Y. 2007, MNRAS, 375, 281

Kazbegi A. Z., Machabeli G. Z., \& Melikidze G. I. 1991, MNRAS, 253, 377

Kazbegi A. Z., Machabeli G. Z., Melikidze G. I., \& Shukre C. 1996, A\&A, 309, 515

Khechinashvili, D. G., \& Melikidze, G. I. 1997, A\&A, 320, L45

Lai, D. 2001, Rev. Mod. Phys., 73, 629

Lai, D., \& Salpeter, E. E. 1997, ApJ, 491, 270

Lomiashvili D., Machabeli G., \& Malov I. 2006, ApJ, 637, 1010

Malov, I, F., \& Machabeli, G. Z. 2002, Astronomy Reports, 46, 684

Malov, I. F., \& Machabeli, G. Z. 2007, Ap\&SS, 29M

Motch, C., Zavlin, V. E., \& Haberl, F. 2003, A\&A, 408, 323

Pavlov, G. G. 2000, Talk at the ITP/UCSB workshop Spin and Magnetism of Young Neutron Stars

Pavlov, G. G., Zavlin, V. E., Trümper, J., \& Neuhäuser, R. 1996, ApJ, 472, L33 Pavlov, G. G., Zavlin, \& V. E., Sanwal, D. 2002, in Neutron Stars and Supernova Remnants, ed. W. Becher, H. Lesch, \& J. Trümper, MPE Report, 278, 273

Pons, J. A., Walter, F. M., Lattimer, J. M., et al. 2002, ApJ, 564, 981

Ransom, S. M., Gaensler, B. M., \& Slane, P. O. 2002, ApJ, 570, L75

Sturrock P. A. 1971, ApJ, 164, 529

Tiengo, A., \& Mereghetti, S. 2007, ApJ, 657, L101

Treves, A., Turolla, R., Zane, S., \& Colpi, M. 2000, PASP, 112, 297

Turolla, R., Zane, S., \& Drake, J. J. 2004, ApJ, 603, 265

Walter, F. M., Wolk, S. J., \& Neuhäuser, R. 1996, Nature 379, 233

Zhelezniakov, V. V., 1977, Volni v Kosmicheskoi Plazme (Moskva: Nauka) 